\title{
ESCEPTICISMO, TEOLOGÍA Y CIENCIA: EL CASO DEL MOVIMIENTO TERRESTRE
}

\author{
Carlos Solís Santos \\ Departamento de Lógica, historia y filosofía de la ciencia, Facultad de Filosofía, Universidad Nacional de Educación a Distancia \\ csolis@fsof.uned.es
}

Recibido: 19 mayo 2014; Aceptado 4 julio 2014.

Cómo citar este artículo/Citation: Solís Santos, Carlos (2015), "Escepticismo, teología y ciencia: el caso del movimiento terrestre", Asclepio 67 (1): p084. doi: http://dx.doi.org/10.3989/asclepio.2015.10

RESUMEN: La tesis de la omnipotencia divina, según la cual Dios puede hacer cualquier cosa que no entrañe contradicción, fue usada por los teólogos bajomedievales como argumento escéptico contra las pretensiones de conocimiento de los físicos. La astronomía, una ciencia matemática, se limitaba a construir modelos de datos respetando los supuestos aceptables por la física que a su vez se debían subordinar a la teología. En el siglo XIV, el teólogo Nicolás de Oresme comparó los argumentos a favor de la rotación terrestre y a favor del giro de los cielos. Siendo un experto matemático y filósofo natural, concluyó la mayor plausibilidad de la primera hipótesis, aunque el escepticismo teológico lo llevó a considerar esas razones insuficientes y a declarar su falsedad por motivos bíblicos. La situación cambió en el siglo XVII. En primer lugar, la Reforma indujo entre los católicos un mayor fundamentalismo; en segundo lugar, los argumentos físicos de Galileo a favor del movimiento terrestre y su refutación del esquema ptolemaico por las fases de Venus hacían insostenible la equidistancia escéptica respecto a ambas posiciones; en tercer lugar, la falta de competencia científica de los actores eclesiásticos llevó a condenar a Galileo y declarar el heliocentrismo falso y formalmente herético.

PALABRAS CLAVE: Movimiento terrestre; Cosmología; Teología; Escepticismo; Omnipotentia Dei absoluta.

\section{SKEPTICISM, THEOLOGY, AND SCIENCE: THE CASE OF EARTH'S MOTION}

ABSTRACT: The Omnipotentia Dei absoluta thesis (any non-contradictory state of fact is possible) was used by theologians as a skeptical argument against any scientific claim unwarranted by biblical exegesis. Mathematical astronomy was bound to build models of data based on physically sound hypothesis acceptable to theology. Fourteenth century theologian Nicolas Oresme weighted the arguments pro Earth and Heavens rotation. Being an expert in mathematics and natural philosophy, concluded the higher plausibility of Earth's rotation, but skeptical considerations declared those arguments insufficient and the opinion false for scriptural reasons. Seventeenth century setting was much different: Reform induced an increase of catholic fundamentalism, while Galileo's physical arguments in support of Copernicanism, together with his refutation of Ptolemaic cosmology due to Venus phases, turned the skeptical balance between both systems untenable. Roman theologians being this time ignoramuses in mathematics and physics, condemned Galileo and declared heliocentrism false, physically absurd, and formally heretic.

KEY WORDS: Earth's motion; Cosmology; Theology; Skepticism; Omnipotentia Dei absoluta.

Copyright: () 2015 CSIC. Este es un artículo de acceso abierto distribuido bajo los términos de la licencia Creative Commons Attribution-Non Commercial (by-nc) Spain 3.0. 
Decía Hegel que los grandes hechos se dan dos veces en la historia y Marx comentaba que «una vez como tragedia y otra como farsa ${ }^{1}$. Pero no siempre es así, sino que en ocasiones ocurre al revés y una comedia se repite como tragedia.

Examinaremos los argumentos escépticos contra el movimiento terrestre de los teólogos Nicolás de Oresme por un lado y de Roberto Belarmino y Urbano VIII por otro, con la finalidad someter la filosofía natural a los dictámenes de los teólogos. La diferente estatura intelectual del primero respecto a los segundos, unida a la amenaza de la Reforma, es lo que convirtió una farsa inocua en una tragedia.

Hacia mediados del siglo XIV, Nicolás de Oresme usó el escepticismo para minar la credibilidad en la ciencia natural y subordinarla a la teología, aunque lo hizo con conocimiento de la ciencia de su época a la que contribuyó notablemente. Planteó la posibilidad del movimiento terrestre sin pretender hallar la verdad, sino «por diversión» (Oresme 1968, p. 538). Pero discutió con penetración los argumentos a favor de la rotación de la Tierra o del giro de los cielos, concluyendo la plausibilidad de la primera hipótesis, aunque acabó rechazándola por mor de la Biblia, según la cual el orbe "está seguro y no se mueve» (Salmos 93.1). Pero casi tres siglos más tarde, el examen del movimiento de la Tierra con nuevos argumentos empíricos y matemáticos por parte de Galileo terminó en tragedia merced a argumentos escépticos y bíblicos de teólogos y papas que poco entendían de cosmología científica.

\section{LA POSIBILIDAD DEL MOVIMIENTO TERRESTRE}

La consideración de la posibilidad de atribuir el movimiento diurno a la Tierra en lugar de a las estrellas fue abordada por Juan Buridán hacia mediados del siglo XIV. En ese momento, no se discutió el movimiento anual en torno al Sol usado por Aristarco de Samos e ignorado por los medievales, sino tan sólo el movimiento de rotación. Los escolásticos conocían el sistema de Heráclides del Ponto², según el cual, la Tierra rota en el centro del universo mientras que el Sol gira en torno a ella con Mercurio y Venus a modo de satélites. En el siglo IX, Escoto Erígena añadió Marte y Júpiter circunsolares. Esta rotación de la Tierra central es la que discutieron los escolásticos parisinos.

Suponer la rotación terrestre implicaba eliminar el movimiento diario al Oeste común a las estrellas fijas y a las errantes (los planetas, el Sol y la Luna). En la astronomía ptolemaica, no menos que en la cosmología aristotélica basada en el primitivo sistema de Eudoxo y Calipo (Metafísica XIII, 8), la esfera de las estrellas gira cada día al Oeste y todos los demás astros errantes tienen también este movimiento común hacia el Oeste; de ahí que salgan y se pongan diariamente ${ }^{3}$. Pero poseen además un movimiento propio al Este, cada uno según un período particular, lo que hace que sus ortos y ocasos vayan variando día a día. Pues bien, lo que plantea Buridán es si se pueden salvar las apariencias suponiendo la rotación diaria de la Tierra hacia el Este y eliminando el movimiento común de todos los demás astros ${ }^{4}$.

Basándose en la relatividad visual, Buridán señala que las apariencias del ciclo nictémero se generan igualmente roten los cielos o la Tierra, siendo un modelo de ello la experiencia de dos barcos que se cruzan en un mar encalmado: "si alguien se mueve en un barco e imagina que está en reposo, al ver otro barco que esté realmente en reposo, le parecerá que el otro barco se mueve ${ }^{5}$. Por supuesto, señala que en el caso de que sea la Tierra y no el cielo la que se mueva, los demás cuerpos celestes mantendrán asimismo sus movimientos propios al Este, más lentos cuanto más alejados del centro, hasta llegar a la esfera de las fijas dotada de la mayor perfección, por lo que le corresponde el reposo. Al mantener los movimientos propios al Este, se producen los diversos aspectos (distancias angulares) que presentan unos respecto a otros (contra la objeción de Alberto de Sajonia). De este modo tanto la rotación celeste como la terrestre cubren deductivamente los mismos fenómenos.

La astronomía era una disciplina matemática que no entendía de materiales ni de fuerzas, sino tan sólo de la construcción de modelos que, mediante movimientos circulares y uniformes, permitiesen deducir los fenómenos astronómicos; esto es, "salvar las apariencias». El asunto de los motores y materiales era competencia de la filosofía natural, por lo que la astronomía debería subordinarse a ella para saber qué supuestos se podían proponer para generar las apariencias ${ }^{6}$. Buridán no se ocupa de astronomía propiamente, sino de cosmología y aporta a favor de la hipótesis del movimiento terrestre un argumento armónico y otro metodológico. El armónico es la disminución de la velocidad de los movimientos propios a medida que se alejan del centro. La Tierra imperfecta se mueve en el centro con máxima velocidad y los astros la disminuyen cuanto más alejados de ella se encuentran, hasta terminar con el reposo de la esfera más noble de las estrellas fijas. Por su lado, el principio de economía aconseja mover menos cosas y menores: más fácil resulta mover a la pequeña Tierra que a los cielos inmensos y, mientras que la Tierra es sólo una, las esferas que se ahorran con su rotación son ocho, pues se eliminan las diarias del Sol, la Luna, los cinco planetas y las estrellas fijas.

No obstante estas ventajas, Buridán señala serias dificultades para la hipótesis, esta vez de naturaleza física ${ }^{7}$. Una de ellas es el argumento tradicional de que si la Tierra rotase, sentiríamos una resistencia del aire mucho mayor que cuando cabalgamos, ya que París se movería a una velocidad enorme ${ }^{8}$. 
No obstante, la objeción se salva suponiendo que la parte baja del aire se mueve con la Tierra y el agua $^{9}$, extremo que elimina asimismo un supuesto calor derivado del movimiento, ya que no es el movimiento en sí quien lo produce, sino la fricción con otro cuerpo en reposo.

Sin embargo, hay un viejo argumento físico contra el movimiento diurno que a Buridán se le antoja incontrovertible. Si se lanza una flecha hacia el zenit, tras el ascenso cae de nuevo a la zona desde donde se lanzó, mientras que si la Tierra se moviese, en el tiempo que tarda la flecha en subir y bajar, el lugar de la Tierra en que se encuentra el arquero se habría desplazado al Oriente una distancia considerable y la flecha caería varios kilómetros al Oeste, cosa que no se observa. La solución anterior, que el aire se mueve con la Tierra, no es aquí suficiente para anular el efecto teórico, ya que en los tiros de flechas con viento lateral, éste arrastra un tanto a la flecha, pero no a su misma velocidad, con lo que debería seguir apreciándose una desviación a Occidente. Téngase en cuenta que la idea de inercia como conservación del movimiento sin necesidad de una fuerza continua no estaba aún disponible y que todo movimiento constante es un efecto que exige una causa constante. De esta manera, la flecha sólo puede acompañar a la Tierra si es arrastrada por el arquero o el aire, y mientras el primero puede hacerlo con eficacia sujetándola rígidamente, el aire sutil sólo ofrece un moderado arrastre, con lo que «el ímpetus violento de la flecha ascendente resistirá al movimiento lateral, de modo que no se moverá tanto como el aire».

En resumen, la experiencia visual no distingue entre ambas posiciones y los argumentos metateóricos de economía y perfección de la quietud favorecen el movimiento terrestre, pero la física de proyectiles refuta la rotación. Es de notar que Buridán, filósofo natural de la facultad de Artes que no pareció interesarse por pasar a la facultad superior de teología, no prestó atención a cuestiones religiosas, cosa que hizo Oresme. Éste fue un notable teólogo y matemático que ahondó en los argumentos de Buridán para concluir con una salida de tono, como si el asunto fuese un pasatiempo sin importancia.

Oresme desarrolla su argumentación científica en tres partes ${ }^{10}$. En primer lugar, arguye que no se puede demostrar con "experiencia alguna» que no se mueva la Tierra, en segundo lugar, que tampoco se puede hacer tal cosa "por razonamiento" y, en tercer lugar, que se pueden aportar razones (persuasiones) a su favor. En los dos primeros puntos Oresme añade novedades respecto al tratamiento del problema por parte de Buridán: una refutación de la objeción física del primero y un tratamiento sensible del espinoso problema de las Escrituras.
Comienza refutando los tres argumentos contrarios basados en la experiencia que discutiera Buridán. El primero, que «nous voions sensiblement» que el Sol, la Luna y muchas estrellas salen y se ponen diariamente (Oresme 1968, p. 520), se elimina recurriendo al modelo de los barcos, pues: «el movimiento local sólo se puede percibir sensiblemente en tanto se vea que un cuerpo se mantiene de manera distinta respeto a otro» (Oresme 1968, p. 522), con lo que la experiencia sólo nos dice que la Tierra y los cielos poseen un movimiento relativo. El segundo argumento del viento del Este se neutraliza asimismo mediante la suposición de que el aire inferior se mueve con la tierra y el agua, usando también el modelo del barco: en la cabina, dónde el aire se halla encerrado, le parecerá al piloto que el aire no se mueve. El tercer argumento que había convencido a Buridán, se refuta ahora originalmente ilustrándolo asimismo mediante el modelo del barco. La idea fundamental es que la flecha posee dos movimientos combinados, uno hacia arriba y otro transversal hacia Oriente. Pero ahora, frente a lo que creía Buridán, no es que el aire arrastre a la flecha (con lo que ésta se hurtaría un tanto a su empuje lateral), sino que ésta "se mueve al Este muy aprisa junto con el aire a través del que pasa y con toda la masa de la parte inferior del mundo, ya descrita, que se mueve con movimiento diurno» (cursivas añadidas) ${ }^{11}$. Por eso la flecha retorna al lugar del que partió, pues todo se mueve a la vez de manera igual. Esto se puede comprobar en un barco que navegue rápidamente al Este: si movemos la mano verticalmente siguiendo el palo, parecerá que traza una línea recta hacia abajo, cuando en realidad se desplaza simultáneamente de manera lateral. Lo mismo pasa con la flecha. Sería un despropósito ver aquí un atisbo de la idea de inercia, pues la Tierra, el aire y la flecha se mueven a Oriente por algún motor no especificado. Oresme no hace un análisis de las causas, sino sólo de los efectos. Pero sin duda considera el conjunto de los cuerpos terrestres como parte de un sistema con movimiento común ${ }^{12}$. Por tanto, frente a Buridán, Oresme concluye que «no puede haber experiencia alguna que demuestre que son los cielos y no la Tierra los que se mueven con movimiento diurno».

Tampoco hay argumentos de razón contra el movimiento terrestre, como que entonces toda la astronomía y buena parte de la física sería falsa. La astronomía se sostiene, ya que los aspectos y movimientos relativos son los mismos en ambos casos. Pero el argumento racional más poderoso, que el físico Buridán no toca pero sí el teólogo Oresme, es que las Escrituras afirman que no se mueve la Tierra ${ }^{13}$. Esos textos se neutralizan señalando que sus expresiones se acomodan al modo de hablar de los humanos, como cuando se dice que Dios se enfada o se aplaca, o que las nubes cubren el cielo (Salmos, 146: 8), cuando es el cielo el que está encima de ellas ${ }^{14}$. 
Finalmente y en tercer lugar, Oresme aduce argumentos que favorecen conceder el movimiento a la Tierra, como el recurso a la simplicidad y economía de Buridán. Recuerda que, según Aristóteles, «Deu et nature ne font rien pour nient», no hacen nada en vano (Oresme 1968, p. 534), por lo que resulta indeseable mover los cielos cada 24 horas, pues de hacerlo así habría que dar a todos los astros dos movimientos contrarios, uno diario al Oeste y otro propio al Este sobre dos ejes diferentes, y además el movimiento común habría de ser rapidísimo. Por el contrario, el movimiento de rotación de la Tierra es sólo uno y menos rápido. Además, si se confiere movimiento a la Tierra, se elimina la novena esfera (la del movimiento diario) dejando tan sólo la octava de un grado al siglo hacia el Este para la precesión de los equinoccios ${ }^{15}$.

Aunque ni la experiencia ni la razón demuestren que se muevan los cielos y repose la Tierra, y aunque haya razones de simplicidad y economía para preferir el movimiento terrestre, todos sostienen, y yo lo creo, que es él [cielo] y no la Tierra el que se mueve: Deus enim firmavit orbem terre, qui non commovebitur, a pesar de las razones en contra, ya que son creencias [persuasions] que no se concluyen de manera evidente. Pero teniendo en cuenta cuanto se ha dicho, se podría creer que es la Tierra y no el cielo la que se mueve, ya que no es evidente lo contrario. No obstante, a simple vista, eso parece tan contrario (o más) a la razón natural como todos o muchos de los artículos de nuestra fe. Así pues, lo que he dicho por diversión [esbatement] puede ser de este modo valioso para refutar y rebatir a quienes quisieran impugnar nuestra fe con razonamientos ${ }^{16}$.

Así pues, la argumentación de Oresme no se orienta a establecer cuál es la constitución del cosmos, sino a mostrar que el conocimiento del mismo es siempre incierto y sólo cabe argüir científicamente de modo inconcluyente, posición escéptica que sirve de paso para la apología de la $\mathrm{fe}^{17}$.

\section{FÍSICA Y TEOLOGÍA}

La diferencia epistemológica de Buridán y Oresme se conecta con sus respectivas áreas profesionales y sus intereses disciplinares. ${ }^{18}$ Hemos visto cómo Oresme, tras exponer con agudeza los argumentos en favor del movimiento terrestre, termina afirmando arbitrariamente que son los cielos quienes giran basándose en un salmo y en la tradición, aunque creyese que en cuestiones naturales las Escrituras se adaptan al modo de hablar común. La finura crítica en cuestiones naturales contrasta con la aceptación simplista de la tradición. No obstante, su escepticismo es genuino, pues tanto el movimiento de la Tierra como el de los cielos no son susceptibles de demostración, sino de persuasión.
Por su parte Buridán, el filósofo natural, diagnostica con agudeza la oposición entre su campo de especialización y el de los teólogos que imponen su autoridad:

... algunas personas, deseando dedicarse a la teología, negaron que pudiéramos tener conocimiento de [fenómenos] naturales y morales. Por ejemplo, no podríamos saber que se mueve el cielo, que el Sol es brillante y que el Sol es caliente, pues tales cosas no son evidentes, ya que Dios podría aniquilar todo eso y no es evidente para ti que quiera o no aniquilarlo, por lo que no es evidente para ti que existan. O bien incluso podría Dios detener el cielo o quitar la luz al Sol o el calor al fuego. Finalmente, dicen que no es evidente que sea de hecho blanca la piedra que ves que es blanca, puesto que incluso sin piedra ni blancura Dios puede crear en tu ojo una imagen del todo semejante a la que ahora tienes del objeto, por lo que juzgarías lo mismo que ahora, a saber, que hay aquí una piedra blanca. De ese modo el juicio sería falso, por lo que no podría ser cierto y evidente $y$, en consecuencia, ni siquiera ahora sería evidente porque no es evidente para ti que Dios lo quiera o no. (Buridán 2000, 8: 44, p.706 y sig. Cf. Klima 2009, Capítulo 12).

La oposición entre física y teología cristalizó en el siglo XIII con la introducción de posiciones escépticas acerca de las verdades demostradas de la ciencia aristotélica. Como es sabido, Aristóteles trataba de exponer como conclusiones necesarias lo que le parecía que era el caso, construyendo un marco teórico en el que pretendía poder encajar cuanto creía saber. Su concepción demostrativa de la ciencia, más propia de la geometría que de la física, consistía en partir de principios indemostrables proporcionados por una inducción infalible ${ }^{19}$, a partir de los cuales se demuestran lógicamente las conclusiones. Obviamente hay poca ciencia efectiva de este estilo en el corpus aristotélico que, en su mayor parte, se dedica a la labor dialéctica de desbrozar el terreno mediante la discusión de opiniones ajenas y la propuesta tentativa de las propias. En cualquier caso, lo que se Ilamó en la Edad Media la demonstratio potissima partía de los principios universales para demostrar silogísticamente la conclusión, mediante una relación causal necesaria entre la esencia de sujeto y el atributo. De este modo, la ciencia de los filósofos naturales entrañaba una necesidad que se imponía a las opiniones de los teólogos.

De ahí que se desarrollasen tesis escépticas acerca de la posibilidad del conocimiento demostrativo, a fin de combatir el determinismo en la interpretación de la naturaleza y de la acción de Dios que llevara a la doctrina averroísta de la doble verdad. Las condenas del obispo Etienne Tempier en París en 1270 y 1277 vinieron a poner fin a la tesis de la doble verdad y al carácter necesario de la ciencia demostrativa, subrayando 
que la omnipotencia absoluta de Dios podría haber hecho cualquier cosa que no entrañase contradicción, de manera que es condenable afirmar la eternidad del mundo, la imposibilidad de su movimiento rectilíneo o de la pluralidad de los mundos, la unicidad del entendimiento, la pluralidad de los primeros motores, etc. (232 en total $)^{20}$. Ciertamente la omnipotentia absoluta se ha limitado a un orden dado concreto (la omnipotentia ordinata), pero tal orden es contingente y no necesario, por lo que no caben demostraciones que coarten la capacidad divina de hacer cualquier cosa no contradictoria. De este modo, demostrar una tesis física (como la rotación terrestre) entrañaría poder probar que su negación ('la Tierra no rota') entraña contradicción, ya que de lo contrario Dios podría haberlo hecho. En cualquier caso, el uso teológico de la omnipotencia sirvió para limitar escépticamente las pretensiones de la ciencia más que para alentar la investigación de lo que de hecho la acción divina ha tenido a bien hacer con su omnipotencia.

Cobró entonces relieve la idea de que las pruebas en cuestiones naturales se reducirían a establecer meras conexiones deductivas, no causales, entre ciertas suposiciones plausibles y sus consecuencias. En 1271, en el momento de las condenas de Tempier, se publicó la traducción de Guillermo de Moerbecke del comentario de Simplicio (siglo VI) al De caelo, lo que puso en circulación la idea de que lo que único que se consigue en física es derivar los fenómenos (salvar las apariencias) a partir de ciertas hipótesis, como en astronomía, "sin afirmar categóricamente que esos diversos mecanismos existen de hecho en los cielos» ${ }^{21}$.

Otros diversos factores contribuyeron al escepticismo en cuestiones científicas al final de la Edad Media ${ }^{22}$, como la postura contraria a la certeza demostrativa expresada en las Summulae logicales de Pedro Hispano, muy leídas desde el siglo XIII al XVI, y especialmente la filosofía de Ockham y el nominalismo que reforzaron en el siglo XIV la oposición de la teología a la presunción de certeza de la filosofía. El empirismo radical y la oposición al descubrimiento de relaciones causales limitaban el conocimiento natural a la intuición de lo singular, lo que por más que permitiese captar la concurrencia de hechos particulares, no justificaba la inferencia de relaciones causales. La ciencia demostrativa de la naturaleza no es posible, ya que las verdades que caracterizan al conocimiento científico sólo se dan en el campo de las matemáticas, la lógica, la teología y la metafísica, que es donde se pueden obtener conclusiones necesarias de premisas evidentes. En la filosofía natural sólo caben enunciados condicionales y meras posibilidades lógicas derivadas de la potentia Dei absoluta.

Por más que a la sensibilidad actual le parezca una buena cosa la negación de verdades incontrovertibles en física y su sustitución por meras conjeturas falibles al gusto de los popperianos, su efecto en las condiciones de la universidad del siglo XIV no fue alentar la indagación natural, sino que llevó a imaginar posibilidades puramente lógicas sin pararse a considerar cuáles son reales (que es lo que les interesará a Copérnico y Galileo unos siglos más tarde), reforzando así el dominio de la teología sobre la ciencia natural. La amenaza de la condena de cualquier afirmación empírica general pendía sobre los físicos. Así vemos a Buridán pedir disculpas por su teoría de que, puesto que la Biblia no dice que los astros sean conducidos por ángeles, sus movimientos podrían explicarse por los impetus que les fueron impartidos por Dios en la creación, los cuales se perpetuarían indefinidamente en un medio sin resistencia: «Pero no lo digo positivamente, sino de modo que pueda recabar de los maestros en teología lo que les quepa enseñarme en estas cuestiones acerca de cómo se producen estas cosas... $\nu^{23}$.

Buridán, el filósofo natural, confía en los sentidos y la posibilidad de la inducción de los primeros principios indemostrables de la ciencia cuando se observa su cumplimiento en muchos casos (in pluribus singularibus), no siendo ninguno negativo. Todo ello bajo la hipótesis de que rige el curso normal de la naturaleza (el impuesto por la omnipotentia Dei ordinata), aunque la absoluta pueda romper ese orden cuando le plazca deteniendo el Sol o convirtiendo el agua en vino. Ex suppositione, pues, «nos es posible comprender la verdad con certeza» ${ }^{24}$.

Aunque Buridán consideraba que el conocimiento natural podía progresar indagando las causas naturales en el curso ordinario de la naturaleza, Oresme tendía a subrayar el engaño de los sentidos hasta el despropósito, como al aseverar la imposibilidad de conocer la posición de los astros por la refracción atmosférica, renunciando a investigarla (Oresme 2007), y al borrar la distinción entre natural y sobrenatural: puesto que sólo hay una verdad y la revelación se conoce mejor que la ciencia, la teología impone sus límites a aquélla. El problema del escepticismo es que acaba incurriendo en la credulidad por falta de criterios epistemológicos. De ahí que los escépticos mitigados (los radicales no escriben ni afirman nada) acaben creyendo cualquier cosa que les guste. El escepticismo de Oresme va acompañado de una credulidad sin cortapisas en la tradición. Por ejemplo, como vimos, el mundo u orbe que no se tambalea de los Salmos es precisamente la Tierra y no el universo, el orbe celeste o cualquier otra estructura que pudiera componer el cosmos; y la ausencia de tambaleo es justamente ausencia de rotación y no de agitación sismológica o de otro tipo. Su manera de argumentar no es consistente, pues frente a las ciencias ocultas, la magia o la astrología, recurre a argumentos científicos y matemáticos, mientras que frente a las ciencias ordinarias esgrime 
argumentos escépticos basados en consideraciones teológicas o epistemológicas (Grant 1988). La magia y la astrología de la que quería defender a su rey no le interesan por diversos motivos particulares ${ }^{25}$. A pesar de que niega las operaciones mágicas suponiendo sin ninguna prueba la existencia de causas naturales para sus portentos, al llegar a las ciencias ordinarias, su escepticismo descree de la posibilidad de llegar nunca a las causas e incluso al conocimiento de los datos, como las razones entre los períodos y distancias de los astros. Su crítica a la astrología servirá de ejemplo ${ }^{26}$.

El análisis clásico de los factores del movimiento de Aristóteles venía a señalar que los movimientos $(V)$ eran directamente proporcionales a los motores $(F)$ e inversamente como las resistencias $(R)$. Esa opinión, en principio plausible, chocaba con el resultado indeseable de que, aunque la resistencia fuese mayor que la fuerza, la razón $F / R$ sería positiva. Para evitarlo, Bradwardine había propuesto una nueva formulación según la cual $F^{\prime} / R^{\prime}=(F / R) v^{\prime} / v$, donde $v^{\prime} / v$ es la razón de las razones $F^{\prime} / R^{\prime}$ y $F / R$. Este es el punto de partida del De proportionibus proportionum, donde Oresme desarrolla con originalidad el problema de relacionar razones con exponentes fraccionarios, racionales o irracionales, explicando el modo de manejar cualquier tipo de magnitudes continuas como velocidades, espacios, tiempos, volúmenes, etc. Una de las proposiciones argumentadas (aunque no estrictamente demostrada) es la Conclusión X de la Parte III que afirma que "Dadas dos razones desconocidas, lo más verosímil es que sean inconmensurables, pues si se proponen muchas [razones] desconocidas, lo más verosímil es que cualquiera sea inconmensurable con cualquier otra». El argumento viene a decir que, a medida que consideremos conjuntos mayores de razones entre enteros (mayores que 1), si tomamos dos de ellas y las relacionamos exponencialmente, aumenta asimismo el porcentaje de exponentes irracionales respecto al de racionales ${ }^{27}$.

El resultado de este argumento es que, dadas dos distancias atravesadas por movimientos continuos cuyas razones son desconocidas, lo más probable es que sean inconmensurables, y lo mismo cabe decir de los tiempos. Por ejemplo, si desconociésemos la razón entre los tiempos de dos movimientos uniformes, lo más probable es que los tiempos de esa razón sean inconmensurables, de donde se sigue que es probable la inconmensurabilidad del día y el año, y lo mismo para cualesquiera pares de movimientos celestes (Oresme 1966, pp. 302-303).

En Ad pauca respicientes, por ejemplo, aplica estas conclusiones a la astrología para demostrar que sus predicciones no pueden ser exactas y que por tanto los astrólogos no pueden conocer el futuro.
No se critica que los astros influyan sobre los acontecimientos terrestres, algo que nadie niega en esta época, sino que estos se puedan predecir. Después de todo, Aristóteles consideraba que las generaciones y corrupciones eran cíclicas (De gen. et corr. 338a4-338b5) y debidas a los movimientos celestes (336b1-24). Pero ahora sabemos que los tiempos y los desplazamientos son probablemente inconmensurables, por lo que los aspectos celestes (oposiciones, conjunciones y demás disposiciones angulares) nunca retornan cíclicamente ni en el mismo punto. Así, la doctrina del Gran año, según la cual cada 36.000 años los astros retornan a las posiciones iniciales reiniciando los cambios cíclicos, no es nada probable. Los eventos celestes, siendo únicos e irrepetibles, no pueden constituir las prótasis de las predicciones astrológicas. Las apódosis pueden derivar de aquellas posiciones, pero la conexión no se puede tabular ni conocer.

No obstante, el requisito de que la recurrencia de los aspectos sea predecible con toda exactitud es una exigencia desmedida. En efecto, desde los caldeos se sabe cómo predecir eclipses de Luna computando las oposiciones del Sol y la Luna (que no tienen por qué ser alineaciones exactas de los centros de los tres cuerpos) y las distancias de ésta al nodo (que no tienen por qué ser nulas). Aun así, los eclipses y las Lunas Ilenas y nuevas se predicen aceptablemente bien con mucha antelación, aspecto que afecta a las mareas y posiblemente a los lunáticos ${ }^{28}$. Este ejemplo simple de cómo los movimientos sinódicos astrales se repiten cíclicamente y producen sus efectos en la Tierra sin precisión matemática absoluta podría ser tomado como modelo de los influjos astrales posibles que, como las mareas, son efectos físicos de posiciones matemáticamente computables con mayor o menor precisión. $\mathrm{Si}$ «la primera parte de la astrología [esto es, la astronomía de posición] es una ciencia especulativa y matemática, muy noble y excelente», pudiendo ser «adecuadamente conocida, aunque no con precisión puntual», no se ve por qué no se podría decir lo mismo de la astrología, al menos por lo que respecta al argumento de la inconmensurabilidad. No es de extrañar que el buen rey de Francia siguiera rodeado de astrólogos y que el arte perviviese aún durante siglos.

Sean cuales fuesen lo motivos ideológicos del rechazo de Oresme del movimiento terrestre, la magia y la astrología, ciertamente estudió profunda y originalmente las cuestiones antes de declarar su opinión. Y si sus compromisos teológicos lo condujeron a negar lo que su razón refrendaba, no dejó de discutir los problemas con pleno conocimiento de causa, algo que no cabe decir de los miembros italianos de la curia a comienzos del siglo XVII. 


\section{BELARMINO Y URBANO VIII SOBRE EL MOVIMIENTO TERRESTRE}

El segundo acto del escepticismo teológico contra la cosmología científica se produjo a principios del siglo XVII, más de dos siglos y medio después, en un contexto diferente no sólo histórico y político, sino especialmente matemático y físico.

En primer lugar, la astronomía copernicana se había presentado como una reforma realista de la astronomía clásica que hasta entonces no había tenido empacho en vadear las consideraciones físicas para proponer construcciones geométricas capaces de generar los fenómenos. Con ello, Copérnico atacaba las licencias geométricas ficticias, pues dar una explicación correcta de los fenómenos, salvar efectivamente las apariencias, dependía de partir de supuestos verdaderos, pues sólo partiendo de la verdad, lo deducido de los principios habría de ser necesariamente verdadero $^{29}$. Por otro lado, los argumentos de los matemáticos (los astrónomos) no se subordinan a la filosofía ni menos aún a la teología, pues la matemática es una disciplina técnica y precisa que es necesario dominar («las matemáticas son para los matemáticos»). De ella depende nuestro conocimiento de la realidad cosmológica y no de los teólogos «charlatanes que, aun siendo ignorantes de todas las matemáticas, presumiendo de un juicio sobre ellas por algún pasaje de las Escrituras» (Copérnico 1973, p. 5), se oponen temerariamente a las conclusiones científicas, como fue el caso de Lactancio, apologista célebre pero matemático nulo, quien se reía de quienes afirmaban que la Tierra era esférica ${ }^{30}$.

En segundo lugar, a principios del siglo XVII, convivían cerca de media docena de esquemas cosmológicos alternativos, cuya comparación y evaluación resultaba insoslayable. La ficción instrumentalista popularizada por Osiander en su prólogo al De revolutionibus, aunque eficaz para paliar las inevitables conmociones físicas, filosóficas y teológicas del copernicanismo, no podía durar mucho. Los sistemas de Ptolomeo, Copérnico y Kepler, más los esquemas de Brahe y otros seguidores de Heráclides, (que nunca pasaron de meras ideas y no dieron lugar a una teoría astronómica operativa) podrían ser geométricamente equivalentes, pero no lo eran físicamente, por lo que el principio de relatividad óptica de Oresme ya no bastaba. Las fases de Venus descubiertas por Galileo mostraban la falsedad de los presupuestos clásicos de la astronomía ptolemaica, mientras que la precisión de la astronomía kepleriana mostraba la superioridad de la ordenación copernicana con los nuevos recursos matemáticos de los movimientos no uniformes y elípticos de sus dos primeras leyes. A partir de la segunda década del siglo, era inevitable la decisión procopernicana de los astrónomos matemáticos sin ataduras ideológicas como las de los jesuitas, pues de ese modo se aclaraba el orden de los planetas, se eliminaban epiciclos y deferentes atribuidos a los planetas interiores y exteriores por el movimiento de la Tierra, y se explicaban trivialmente muchos detalles misteriosos de los modelos ptolemaicos, como la retrogradación de los planetas exteriores en la oposición y la dependencia de la Luna y todos los planetas del Sol ${ }^{31}$. Y no sólo eso, sino que el reto copernicano alentó el desarrollo de una nueva física inercial capaz de acomodar el movimiento terrestre y el comportamiento de nuestros graves y proyectiles, tarea en la que destacó Galileo, quien además explicó algunos efectos observables como resultado del movimiento terrestre ${ }^{32}$. Con ello, el problema no era ya el de dar explicaciones causales necesarias (demonstratio potissima) de los fenómenos, sino comparar cómo resolvían los problemas las diversas cosmologías para evaluar cuál era superior.

La situación científica y epistemológica era radicalmente nueva, pero las autoridades eclesiásticas que intervinieron no eran ya escolásticos eruditos, duchos en matemáticas y filosofía natural no menos que en teología, como Oresme o Zúñiga, sino aficionados a la cultura humanística superficial, como Maffeo Barberini, o teólogos fundamentalistas sin formación matemática ni ganas de adquirirla, como Roberto Belarmino.

\section{ASTRONOMÍA BÍBLICA Y ESCEPTICISMO CIENTÍFICO}

Galileo expresó su convicción copernicana al final de La gaceta sideral de 1610 y en varios lugares de la Historia y demostraciones sobre las manchas solares de 1613 (Opere III-1, p. 95; V, pp. 99, 195, 238). La defensa del copernicanismo contra lo que parece decir la Biblia aquí y allá llevó en Febrero y Marzo de 1615 a las denuncias de Lorini y Caccini ${ }^{33}$. La de este último llevó su tiempo y al cabo de un año, el 23 de Febrero de 1616, una comisión de once consultores teólogos sin mayores conocimientos de astronomía dictaminó que la opinión copernicana era científicamente estúpida y absurda ("stultam et absurdam in philosophia»), la tesis de quietud del Sol era formalmente herética y la del movimiento de la Tierra, «errónea en la fe» (Opere XIX, p. 321). Que teológicamente el copernicanismo contradiga o no la letra de la Biblia no nos preocupa ahora tanto como la declaración sin argumentos de que es científicamente falsa, como se publicó finalmente en el Decreto del 5 de $\mathrm{Marzo}^{34}$. En este momento no se condenó a Galileo, una figura famosa y reverenciada en toda Europa, sino sólo el copernicanismo. También se prohibieron tres libros que lo defendían, unos hasta su corrección (el de Copérnico y el de Zúñiga) y otro sin paliativos (la Carta de Paolo Foscarini sobre la opinión de los pitagóricos 
y de Copérnico). No se mencionaron explícitamente las obras de Galileo, pero "se prohíben, condenan y suspenden» todos los demás libros que digan lo mismo. Un par de días después del veredicto de los consultores, el Papa ordenó a Roberto Belarmino que advirtiera a Galileo del dictamen y de que por tanto debía abandonar el copernicanismo, cosa que hizo el día 26 de Febrero, sin que Galileo protestara y sin que personalmente tuviera que abjurar ni se le impusiera penitencia alguna (Opere XIX, pp. 321-22; Belarmino 1984, p. 25).

El Decreto no explica las razones por las que el copernicanismo es falso, pero Belarmino, cardenal miembro del Santo Oficio y consejero teológico de la Santa Sede, había señalado algunas pistas un año antes. El 12 de Abril de 1615 escribió una carta a Foscarini ${ }^{35}$ en la que señalaba que él y Galileo deberían usar la hipótesis copernicana como una suposición útil y no como una verdad, tal como hiciera Copérnico, porque de esa manera "se salvan mejor las apariencias que con excéntricas y epiciclos ${ }^{36}$. Con ello muestra no haber leído a Copérnico, pues parece creer que las excéntricas y epiciclos son cosa de Ptolomeo, cuando, como señala Galileo, Copérnico también los usa. Su contraposición de Ptolomeo con Copérnico no es cualificada. Tras afirmar que todo lo que dice la Biblia es verdad, aunque no se trate de cuestiones de fe y costumbres, ya que lo dice Dios, señala un tanto incongruentemente que si alguna vez se diese una demostración verdadera del movimiento terrestre, entonces ya verían los teólogos qué hacer con las Escrituras. Pero no cree que ello sea posible, ya que de que el copernicanismo explique los hechos no se sigue que sea verdad, por lo que haría falta una demostración directa por otros medios. Obviamente no hay demostraciones de ese estilo en la ciencia empírica. Galileo respondió a este reto añadiendo a las razones matemáticas nuevas pruebas físicas del movimiento terrestre con su teoría de las mareas. Pero, como bien señala en el comentario a la carta, su postura como científico no es que se crea que el esquema copernicano es la verdad, sino que no se le niegue lo que se concede al geocentrismo: la posibilidad de discutirlo para comparar los méritos relativos de ambos. La pretensión de que Belarmino y Pio $\mathrm{V}$ se pusieran a estudiar astronomía no era muy realista, pero ciertamente no era justo exigir al heliocentrismo una demostración positiva (aparte de su capacidad explicativa de los hechos) mientras se condescendía con el ptolemaismo que había sido refutado por las fases de Venus. Que las consecuencias del heliocentrismo sean ciertas no es una demostración de que la teoría sea verdadera, pero «es aún más cierto que el otro sistema ordinariamente aceptado ni siquiera es capaz de dar razón de las apariencias». No necesitaba leer a Popper para saber que el geocentrismo "es sin duda falso, del mismo modo que está claro que éste [el heliocentrismo], que se corresponde muy bien [con las apariencias], podría ser verdadero» (Opere V, p. 369; itálicas mías).

Belarmino ya había hablado de cosmología al comentar los seis días de la creación en sus Lectiones Lovanienses, dictadas de Octubre de 1570 a la Pascua de 1572. Pero no trató las cuestiones cosmológicas mediante matemáticas y observaciones, sino citando la Biblia que en realidad no se ocupa de la constitución del cosmos, aunque diga de pasada cosas más o menos peregrinas al respecto. Eso lo llevó a desestimar a Aristóteles y a distinguir tres partes: la atmósfera terrestre, el cielo de los astros y el cielo empíreo de los bienaventurados. Rechazó la teoría aristotélica del éter incorruptible por las alusiones bíblicas a un único cielo ígneo y fluido ${ }^{37}$ en el que los astros vagan libremente «con movimiento propio, como aves por el aire o peces por el agua» (Belarmino 1984, p. 19), sin mecanismo alguno que explicase sus movimientos ${ }^{38}$. Desde los tiempos de Platón, la astronomía había consistido en reducir los movimientos irregulares de los astros a combinaciones de movimientos uniformes y circulares fáciles de describir y computar matemáticamente. La idea de Belarmino de que los únicos movimientos reales de los astros son los aparentes significaba renunciar a la astronomía. Por ejemplo, tradicionalmente el movimiento diario de las estrellas al Oeste se explicaba mediante la octava esfera que giraba uniformemente en torno a los polos celestes, mientras que la precesión de los equinoccios se acomodaba por el movimiento regular de otra esfera con un movimiento de casi un grado al año ${ }^{39}$. La ocurrencia de Belarmino de que las estrellas nadan o vuelan libremente sin ser movidas por una esfera de éter, planteaba serios problemas a la hora de explicar por qué giran todas en líneas paralelas al ecuador celeste y con velocidades decrecientes en función de la distancia al polo de giro, como si en realidad estuviesen fijas en una esfera ${ }^{40}$. Por otro lado, no veía la necesidad de introducir otra esfera para la precesión porque su período de entre 36.000 y 49.000 años (según el cálculo ptolemaico y el alfonsí respectivamente) le parece «ridículo e increíble» porque «el mundo no va a durar tanto ${ }^{41}$.

Los problemas que plantean las estrellas errantes son todavía mayores. Tradicionalmente se suponía que los astros errantes (los planetas, el Sol y la Luna) participaban del movimiento diario y regular de las fijas, al que cada uno añadía su movimiento propio al Este. El Sol caminaba casi un grado diario al Este, recorriendo los signos. La idea de Belarmino es eli- 
minar la composición de movimientos y decir que el Sol camina al Oeste como las estrellas sólo que un poco más lentamente, con lo que se retrasa respecto a las fijas, idea ya refutada en el Almagesto (Ptolomeo 1984, I.8, p. 46) que no parece haber leído, ya que el movimiento diario y anual se realizan sobre ejes distintos. Además el Sol recorre la Eclíptica más rápido por el invierno, de manera que los astrónomos no se conformaban con el Sol medio y preferían explicar el movimiento solar mediante una excéntrica o un pequeño epiciclo. De esta manera, computando los movimientos de las diversas esferas, se podía predecir la posición solar en el futuro, y combinando esos movimientos con los de los diversos círculos de la Luna, se podían predecir los eclipses lunares. Con astros nadando libres, nada hay que computar. Por otro lado, como el Sol y en gran medida todos los demás planetas, camina por la Eclíptica, que gira aparentemente con las estrellas, Belarmino supone que el Sol y los planetas trazan espirales a lo largo del año, sin que le preocupe cómo explicar esta conducta común ${ }^{42}$.

Si consideramos los movimientos aparentes de los cinco planetas, las cosas empeoran, pues sus movimientos son más irregulares que los de las luminarias y presentan estacionamientos y retrogradaciones, esto es, avances hacia Occidente contra el trasfondo de las fijas, cuando normalmente van hacia el Oriente. Si decimos que se mueven a Occidente ora más despacio que las fijas, ora más aprisa, sin ofrecer una explicación matemática, se acaba la teoría planetaria y la física celeste, pues nada explicaría que los planetas exteriores aceleren en la oposición y los interiores, cada dos conjunciones. Eso es algo que Ptolomeo hacía a su modo mediante un epiciclo, exigiendo que el radio del epiciclo de los exteriores fuese paralelo a la dirección al Sol medio y, en el caso de los interiores, que el centro del epiciclo estuviese en la dirección al Sol. Copérnico, por supuesto, lo explica por el movimiento terrestre. Belarmino no dice nada de las retrogradaciones y difícilmente podría convencer a ningún científico de que siguiese su camino impracticable, consistente en aceptar como reales los movimientos aparentes que no resultan computables $^{43}$. Obviamente le importaban más sus lucubraciones filológicas sobre expresiones oscuras de la Biblia que el estudio de la naturaleza.

En las Lectiones Lovanienses no aparece Copérnico y es muy posible que no hubiera oído hablar de él ${ }^{44}$. Pero en 1615 , tras la defensa galileana del heliocentrismo y sin estudiar el tema, Belarmino no dudó en decirle lo que tenía que creer y lo que podía defender. El contraste de Belarmino con los teólogos escolásticos como Oresme o Zúñiga, que sabían de qué hablaban, no puede ser más llamativo. Aunque Oresme acabe creyendo en la inmovilidad de la Tierra, su conocimiento de los argumentos en pro y en contra es notable; por el contrario, la ignorancia matemática de Belarmino sólo es comparable a su seguridad en una pseudociencia inconcluyente ${ }^{45}$. Él lo sabe, pues ya señalara en 1570 que no es tarea del teólogo ocuparse de astronomía, pero como hay discrepancias entre los astrónomos, podemos seleccionar la teoría más concorde con las Escrituras. No obstante, si se tornara evidente que se mueven los cielos y no los astros, «entonces ya se verá cómo interpretar las Escrituras» ${ }^{46}$. Repite lo mismo en 1615 (Opere XII, p. 172). Aunque ni él mismo está seguro de sus elucubraciones escriturísticas, ello no le impidió amonestar a Galileo que sí sabía de qué hablaba. La cuestión no era de argumentos sino de quién debe subordinarse a quién.

\section{EL ARGUMENTO ANGÉLICO}

En 1621 Belarmino se fue al cielo a averiguar cómo se mueven los astros y un par de años más tarde ascendió a la sede pontificia Urbano VIII que no era teólogo ni matemático, pero amaba la cultura mundana. Gracias a él, Galileo se decidió a defender el copernicanismo en un Diálogo sobre el flujo y reflujo del mar que creía poder derivar del doble movimiento terrestre. No obstante, se le prohibió mencionar las mareas en el título y se le impuso un prólogo escéptico en el que se decía que ningún experimento podría decidir sobre el movimiento o quietud de la Tierra, opiniones ambas que el Diálogo debía tratar «de manera inconcluyente».

Este escepticismo impuesto remitía a la potencia absoluta divina, un argumento del que el papa estaba muy orgulloso y que conocemos por su teólogo personal, Agostino Oreggi, a quien Ilamaba «il mio Bellarmino». Como el difunto Roberto Belarmino, Oreggi escribió sobre la creación basándose exclusivamente en las Escrituras y la patrística, subrayando asimismo la incertidumbre del conocimiento físico, la seguridad del teológico y los límites que éste impone a la ciencia (De opere sex dierum, 1632; cf. Bianchi 2001, p. 580). El argumento hubo de introducirse al final del Diálogo por orden expresa del Maestro del Sacro Palazzo, aunque el que apareciera en boca de Simplicio no gustó nada (Opere XIX, p. 226). El argumento viene a decir que, puesto que la potencia divina es infinita, un efecto dado puede deberse a mil causas que ni siquiera imaginamos, por lo que la ciencia nada puede afirmar.

Los tres grandes argumentos de Galileo a favor del movimiento terrestre eran las retrogradaciones y variaciones de distancia de los planetas (Opere VII, pp. 
369-372), el patrón estacional del movimiento de las manchas solares (374-381) y las mareas y los alisios (450-466). Se trata de tres tipos de efectos derivados del movimiento terrestre. Pero esos efectos no demuestran nada si no queremos cometer la falacia de afirmación de consiguiente y negar que Dios pueda hacer lo mismo de otra manera. Por ejemplo, en el caso de las mareas, no cabe dudar de que "Dios con su infinito poder y sabiduría podría conferir al elemento del agua el movimiento recíproco que comprobamos en él de una manera distinta que moviendo el vasto recipiente». Negarlo sería «limitar y coartar la potencia y sabiduría divinas a una fantasía particular suya» (Opere VII, pp. 487 y 488).

El argumento se amplía un poco en la noticia que da de él Oreggi. El papa, cuando todavía era cardenal, se lo habría expuesto a un Galileo que pretendía explicar muchos fenómenos con la movilidad terrestre. Le preguntó si Dios no podría hacer que ocurrieran esas cosas con otra disposición de los astros y, sin esperar una respuesta, arguyó que «si dices que no, deberías probar que implica una contradicción que eso pueda suceder de un modo distinto a como lo has ideado, pues con su potencia infinita Dios puede todo cuanto no implica contradicción ${ }^{47}$

Según esto, sólo se podrían sostener proposiciones formales, como que los ángulos de un triángulo midan dos rectos, cuya negación entraña contradicción, pero no hay cabida para la ciencia empírica. Lo malo de este llamado argumento es su excesiva potencia, pues puede aplicarse a cualquier afirmación empírica. El argumento angélico es como la hipótesis de un genio maligno que puede engañarnos siempre, sin que haya refugio en el cogito o en la bondad divina. No obstante, Galileo busca salir de las garras de la omnipotencia recurriendo a una imagen racional de la acción divina basada en la simplicidad. Contestando a algunas críticas de Morin tendentes a justificar cualquier posibilidad anticopernicana por recurso a la omnipotencia (Dios podría hacer girar la enorme esfera de las fijas en un minuto, pues «quod qui negat, Dei omnipotentiam negat $\left.{ }^{48}\right)$, señala que:

no investigamos lo que podría hacer, sino lo que ha hecho..., viendo que en sus operaciones tiende siempre a los procedimientos más fáciles y simples, por más que su potencia se manifestaría mejor en los más difíciles.

Por ejemplo, podría hacer los huesos de las aves de oro macizo, su carne más pesada que el plomo, con alas diminutas y con las venas llenas de mercurio, con lo que su omnipotencia brillaría más al hacerlos volar; sin embargo ha decidido hacer "los huesos, la carne y las plumas muy ligeras... para enseñarnos que gusta de la simplicidad y facilidad» (Opere VII, pp. 565-566).
Su oposición al argumento angélico queda patente en el Diálogo cuando, ante la declaración de Sagredo de que los argumentos favorables al movimiento terrestre son manifiestamente concluyentes, Salviati dice, siguiendo las indicaciones de los censores, que su intención no era resolver la cuestión del movimiento terrestre, sino "proponer las razones naturales y astronómicas» a favor de ambas posiciones, dejando a cada cual sacar la conclusión pertinente. Pero se desdice inmediatamente al señalar que "ésta al final no habría de ser ambigua dado que, siendo necesario que una de las dos constituciones sea necesariamente verdadera y la otra necesariamente falsa, es imposible que (manteniéndonos en los límites de las doctrinas humanas) las argumentaciones aducidas por la parte verdadera no se manifiesten tan concluyentes como vanas e ineficaces las contrarias» (Opere VII, p. 383). En una palabra, no sólo confiamos en el principio de simplicidad en la naturaleza, sino que ante dos posiciones contradictorias (la Tierra se mueve/no se mueve), la falsedad de una entraña la verdad de la otra.

Como se sabe, eso le costó la condena y encarcelamiento de por vida. Obviamente, el buen papa no aplicaba su argumento al geocentrismo, a la separabilidad del alma del cuerpo o a cualquiera otra de sus doctrinas predilectas, sino tan sólo a las opiniones novedosas que no encajaban con aquéllas. Por eso no puede considerarse que el argumento angélico sea una tesis epistemológica acerca de la ciencia (Bianchi 2000 , p. 244). Como se ha visto, el viejo argumento de la omnipotencia divina servía para atacar cualesquiera novedades que pusiesen en entredicho las supuestas evidencias de los teólogos: en el siglo XIII sirvió para oponerse a la filosofía de Aristóteles y en el siglo XVII, tras su adopción por la teología católica, para defenderla frente a las novedades copernicanas.

$$
* * *
$$

Los escolásticos del XIV consideraron con honestidad intelectual las razones sobre el movimiento terrestre o celeste, las discutieron con los mejores argumentos y, ante la plausibilidad de ambas posturas, Oresme optó cómodamente por la posición acorde con la interpretación tradicional de las Escrituras ${ }^{49}$. Todo parecía un juego intelectual sin grandes riesgos. Pero la comedia se tornó en tragedia cuando los argumentos físicos y astronómicos a favor del heliocentrismo debidos a Galileo obligaron a tomar una decisión teórica que los teólogos de la curia no estaban científicamente preparados para abordar. No estando dispuestos a volver a la facultad de Artes ni a abandonar la posición de preeminencia que la teología, el saber en el que habían invertido sus años de estudio, pretendía tener sobre la ciencia natural, optaron por el camino más cómodo que les permitía su posición social: la condena de la ciencia y del científico. 


\section{NOTAS}

1 Al comienzo de Marx 1852.

2 Refutado por Ptolomeo en el Libro I.7 del Almagesto (Ptolomeo 1984, pp. 44-5) y difundido gracias a la noticia de Simplicio en sus In de caelo commentaria, traducidos en 1271 por Guillermo de Moerbecke; cf. ad II.8 (289b1), II.13 (293b30) y II.14 (297a2).

3 Aristóteles lo asegura allí confiriendo a cada astro errante un sistema de esferas, la primera de las cuales tiene el mismo movimiento que la de las estrellas fijas.

4 Alberto de Sajonia no pareció entender bien este planeamiento, pues incluye en el reposo celeste la eliminación de los movimientos propios, con lo que no se salvan los fenómenos sinódicos, eclipses, conjunciones y oposiciones: "nullo modo per motum terre et quietem celi possemus salvare opositiones et coniuntiones planetarum, nec eclipses solis et lune" (Clagett 1959 , p. 586 nota).

5 Buridán 1942, II. 22, p. 227. Ya había dicho Ptolomeo que "no hay quizá nada en los fenómenos celestes que vaya contra esta hipótesis" (Ptolomeo 1984, libro I. 7, p. 45).

6 Lo hizo Ptolomeo al empezar el Almagesto (Ptolomeo 1984, Libro I, 3-8), con la discusión de la forma y movimientos de Tierra y cielos.

7 Antes ha eliminado un par de objeciones inanes. La primera, el recurso a la autoridad de Aristóteles y los astrónomos ptolemaicos, señalando que la autoridad no constituye un argumento. La segunda, la observación ingenua de que "aparece a los sentidos que las estrellas se mueven de este a oeste" (esgrimida más adelante por Roberto Belarmino, véase la nota 43), que contrarresta señalando que se ve lo mismo muévase la Tierra o los cielos.

8 Buridán no echa las cuentas, pero los matemáticos de la época de Aristóteles calculaban la circunferencia terrestre en unos 400.000 estadios (De caelo, II.14, 298a15), mucho más que los 240.000 o 250.000 de Posidonio y Eratóstenes, lo que a la latitud de París daría una velocidad de unos $1.650 \mathrm{Km} / \mathrm{h}$.

9 Opinión retomada por Copérnico en el De revolutionibus, I. 8, y adaptada por Galileo para los alisios; cf. Solís 2001, p. 370 y sigs.

10 Oresme 1968, II. 25 , pp. 520-538.

11 “...la seëtte traite en haut, aveques ce trait est meue vers orient tres isnelement aveques l'aer parmi lequel elle passe, et aveques toute la masse de la basse partie du monde devant designee qui est meue du mouvemet journal" (Oresme 1968, p. 524).

12 La analogía de la mano del piloto y la flecha resulta dinámicamente oscura. La mano está rígidamente unida al cuerpo que se afinca en la cubierta del barco, que es el que arrastra consigo al piloto; pero la flecha no está rígidamente unida a nada, por lo que queda en el aire (nunca mejor dicho) cual sería la causa de su movimiento lateral, y de ahí la tentación de proyectar sobre el argumento una ley de inercia. Sin embargo, los escolásticos del XIV nunca pusieron en tela de juicio que un movimiento constante exija la acción de una fuerza constante, lo que plantea un problema con la flecha. La solución de Buridán es suponer que el motor inicial en contacto con un proyectil le comunica una fuerza o cualidad incorpórea, el impetus, capaz de seguir moviendo al móvil según la misma trayectoria del motor (arriba, abajo o en círculo) hasta que la resistencia (el medio o la gravedad) la gaste. En los cielos perfectos, donde no hay resistencia, el impetus nunca se agota. Oresme sigue a Buridán con algunas diferencias, como que el impetus se agota espontáneamente sin necesidad de resistencia y la renuencia a extrapolar la doctrina de impetus del mundo terrestre al celeste, pues el movimiento de los astros podría ser voluntario. Cf. Anneliese Maier 1955

13 Oresme parece aludir a Eclesiastés I: 3 ("El Sol sale y se pone, corre a su lugar y allí vuelve a salir"); a Salmos 93: 1 ("el orbe está seguro, no vacila"), donde lo de "orbe" se interpreta como "Tierra" y no vacila como "no se mueve", y Josué 10:12, donde se detiene el movimiento del Sol. Hay otros sitios en los que la Tierra se agita, como Joel 4: 16, pero eso no se interpretó favorablemente al movimiento terrestre; o el famoso de Job IX, 6 ("Él mueve la Tierra de su sitio") en que se basó Diego de Zúñiga (1584, p. 205 y sig.; Blackwell 1991, pp. 185 y sig.) para defender la compatibilidad de la Biblia y la astronomía copernicana.

14 La Biblia "se conforme en ceste partie a la maniere de commun parler humain... si comme la ou il est escript que Dieu se repenti..." (Oresme 1968, p. 530).

15 Oresme 1968, p. 524. El mundo medieval constaba de ocho esferas (siete para las estrellas errantes y una octava para las fijas). Por encima de ellas estaba la décima esfera del cielo empíreo (creado el primer día como habitat de los ángeles) y la novena esfera del cielo cristalino (las aguas superiores de Génesis I. 7). Como las fijas presentaban tres movimientos, el diario, la precesión y la trepidación (un movimiento espurio derivado de datos incorrectos que Oresme ignora aquí), se tendió a atribuir uno a cada una de las tres esferas superiores; por ejemplo, Alberto de Sajonia prescindió del cielo empíreo inmóvil y mantuvo tres esferas para las estrellas, confiriendo el movimiento diario a la decima, el de precesión a la novena y la trepidación a la octava.

16 Oresme 1968, pp. 536 y 538. En las Questiones de spera, escritas al comienzo de su carrera, discutía las objeciones al movimiento diurno para terminar declarando que la verdad es que no se mueve la Tierra sino los cielos, aunque esto tampoco se puede demostrar, sino tan solo es dado argüir su plausibilidad: "Dico tamen quod conclusio non potest demonstrari sed persuaderi, sicut patuit ex positione oposita et ideo est credita" (Clagett 1959, p. 609n)

17 Sobre el escepticismo de Oresme y sus posiciones epistémicas, véase Grant 1988 y López Prada 2014.

18 Sobre las diferencias epistemológicas de Buridán y Oresme enraizadas en sus intereses disciplinares en la física y la teología respectivamente, véase Grant 1993, pp. 103-5; López Prada 2014, §1.3.

19 En los Segundos analíticos, II. 19, 100a 15-100b 5, se explica la capacidad humana de partir de la sensación, pasar a la memoria y de ahí a la experiencia acumulada que es capaz de estabilizar lo universal en el alma: "cuando se ha establecido un 
cierto número de particulares lógicamente indiscriminables, está presente en el alma un universal primitivo, pues aunque se perciba lo particular, su contenido es universal [...] Así está claro que resulta necesario que nos familiaricemos con los primeros principios por inducción, pues la percepción infunde lo universal de ese modo".

20 Trece en 1270 y 219 en 1277; cf. Denifle 1889, pp. 486-7 y 543-8. Piché 1999 arguye que la condena revela no tanto discrepancias doctrinales cuanto el poder institucional de subordinar a la teología la racionalidad humana de los maestros de artes. Sobre las omipotencias cf. Funkenstein 1968, cap. 3.

21 Ad De caelo III. 1, donde Aristóteles critica la composición de los cuerpos a base de figuras geométricas desarrollada en el Timeo. Simplicio apunta que quizá Platón no considerase que los cuerpos fuesen realmente una combinación de triángulos, aunque suponerlo le servía para derivar los hechos. Contra el sentir de Pierre Duhem, esta posición convencionalista unida a las condenas de Tempier no contribuyeron a desarrollar la ciencia, sino a minar la confianza en el saber humano. "The philosophers, or masters of arts, may have found the condemnations burdensome, but the theologians found it a convenient instrument with which to humble the philosophers and their reliance on natural reason" (Grant 1962, p. 200).

22 Grant 1962, p. 201-207. Sobre el método científico en los escolásticos tardíos, véase Crombie 1959, Vol. II, Capítulo I. 1.

23 Quaestiones super octo physicorum libros Aristotelis, Libro VIII, Cuestión 12. Clagett 1959, p. 536.

24 In metaphysicen Aristiotelis quaestiones argutissimae, libro II, cuestión 1. Grant 1993, pp. 88-89.

25 La magia podría ser fuente de milagros, dado que cree en el diablo; pero como eso crearía confusión con los milagros de la omnipotencia divina, un poco de racionalismo y recurso a causas naturales no está de más: "si la fe no afirmara su [de los demonios] existencia, no podría probarse por ningún efecto natural, pues todo se puede explicar naturalmente (Oresme 1972, quodlibet 28). Por su lado, la astrología compromete el libre arbitrio y alienta la influencia de los astrólogos más bien que de los eclesiásticos sobre el monarca, por lo que la ataca con argumentos tan precisos como ineficaces, como se verá.

26 Dedicó a ello varios tratados, como el Livre de divinacions y el Tractatus contra iudiciarios astronomos et principes in talibus se occupantes (1952), Ad pauca respicintes (1966), Questio contra divinatores horoscopios (1976), Tractatus de commensurabilitate vel incommensurabilitate motuum celi (1971) y otros relacionados, como De visione stellarum (2007) y Quodlibeta (1973), así como una supuesta traducción suya del Quadripartitum (el Tetrabiblos) de Ptolomeo. No se puede decir que no supiera de qué hablaba. Para los escritos de Oresme, véase Weijers 2005, pp. 175-191; Clagett 1968, pp. 645-648.

27 Cf. Oresme 1966, p. 247. Para cualquier conjunto de razones n/1 (con $n=2,3 \ldots$ ), la fracción de razones de razones irracionales respecto a las racionales para cada pareja crece a medida que aumenta el conjunto considerado. Así, de las 4.950 razones de razones (con el denominador menor que el numerador) entre parejas de las cien razones de $2 / 1$ a $101 / 1$, sólo 25 son racio- nales y 4.925 irracionales. Es un argumento similar al esgrimido por Galileo para la mayor frecuencia de raíces que de cuadrados a medida que tomamos conjuntos mayores de naturales a partir de la unidad, aunque si se consideran conjuntos infinitos, ambas frecuencias se igualan. Galileo, Opere, VII, pp. 78-79.

28 Véase en Oresme 1966, p. 304, el carácter único e irrepetible de un posible eclipse total de Luna, incluso en toda la eternidad, lo que no está probado. En cualquier caso, la astronomía se hurta a los peligros de la inconmensurabilidad porque una razón incognoscible se puede acotar con dos lo bastante próximas, "Et hoc debet sufficere" (pp. 288-291), lo que podría salvar no sólo la astronomía, sino también la astrología.

29 Mientras que las teorías homocéntricas (como las de EudoxoCalipo) no predicen los hechos, las que recurren a excéntricas y epiciclos pueden hacerlo, pero al precio de introducir expedientes (los ecuantes) que contravienen los principios de regularidad del movimiento, con lo que su astronomía es un monstruo incapaz de mostrar el orden y simetría de las partes del mundo, como la correspondencia entre distancias, períodos y velocidades: "Si las hipótesis supuestas por ellos no fuesen falsas, todo lo que de ellas se deduce se podría verificar sin lugar a dudas" (Copérnico 1973, II, 4).

30 En sus De divinis institutionibus III, 24, sobre los antípodas, se lee: "Aut est quidem tan ineptus qui credat esse homines quorum vestigia sunt superiora quam capita?... pluvias et nives $\&$ gradinem sursum versus cadere in terram?” (“¿Acaso habrá alguien tan inepto que crea que haya hombres con los pies por encima de la cabeza?... ¿que las Iluvias nieves y granizo caigan en la tierra hacia arriba?"). Este pasaje del De revolutionibus fue suprimido en la corrección del libro de 1620, a consecuencia del Decreto contra el copernicanismo del 5 de Marzo de 1616, junto con el capítulo 8 del libro I, en el que se refutan los argumentos físicos contra el movimiento terrestre, y diversas afirmaciones aisladas que mencionan el movimiento de la Tierra; Galileo, Opere XIX, p. 400.

31 Sin saber la razón de ello, el Almagesto incluye el año solar (debido en realidad al movimiento terrestre) como el único parámetro numérico común a todos los modelos, razón por la cual empieza con la teórica solar (Ptolomeo 1984, libro, III).

32 Especialmente los alisios (que se deben al movimiento terrestre por mecanismos distintos de los estimados por Galileo), las mareas (que no se deben realmente al movimiento terrestre) y el patrón estacional del movimiento de las manchas solares, fácilmente explicable en el esquema heliocéntrico pero dinámicamente inverosímil en la perspectiva geocéntrica. Sea cual sea la explicación actual, los tres eran argumentos poderosos.

33 La obra de referencia sobre la Iglesia y Galileo es Beltrán 2006.

34 Opere XIX, p. 323, donde se tilda al copernicanismo de "doctrina pitagórica falsa y totalmente contraria a las Sagradas Escrituras".

35 Opere XII, pp. 171-72. Véanse los comentarios de Galileo, Opere V, pp. 367-370.

36 Es improbable que Belarmino no supiera que Copérnico no era instrumentalista, sino plenamente realista; cf. Beltrán 206, pp. 692-694. 
37 Siete meses más tarde, la observación de una nova confirmó casualmente la idea de que en los cielos había generaciones y corrupciones. Asimismo, el paso del cometa de 1577 a través de las órbitas de los planetas ponía en tela de juicio la existencia de orbes sólidos que los arrastrasen. Ambas novedades fueron explotadas por Tycho Brahe contra Aristóteles.

38 Según comunicaba Grienberger a Biancani, Clavio, maestro de los matemáticos jesuitas, "hasta el final de sus días aborreció la liquidez de los cielos" y de paso el sistema de Tycho Brahe, ya que eliminaba las esferas sólidas tradicionales y privaba a los astros de una razón matemáticamente descriptible de sus movimientos regulares. Cf. Lattis 1994, pp. 201-202.

39 La precesión fue descubierta por Hiparco en el siglo II a.C. comparando el año sidéreo (respecto a la eclíptica) con el trópico (de retorno a un equinoccio) que resultaba más corto. Si el equinoccio retrocede respecto al orden de los signos, el Sol llega a él antes que a la misma longitud eclíptica. Debido a que se hallaron luego otros valores para la precesión, se supuso que la velocidad variaba y Thabit ibn Qurra ingenió un sistema que explicaba esta supuesta variación con el movimiento uniforme de otra esfera.

40 En el Almagesto (Ptolomeo 1984, libro I. 3) se muestra por qué las estrellas están en una esfera, pero Belarmino no parece saberlo; cf. Belarmino 1984, pp. 18-20. Se basa para decir que las estrellas no están fijas en una esfera celeste en que el Génesis dice que Dios colocó los astros en el cielo y al hombre en el Paraíso; pero así como éste no quedó fijo al suelo, las estrellas tampoco quedaron fijadas al cielo. Otros jesuitas como B. Pereira y C. Clavio criticaron estas ideas de Belarmino; cf. Clavio 1611, pp. 2-24, 299; Belarmino 1984, p. 38, n. 88; Lattis 1994, pp. 97-102; Bianchi 2001, nota 33.

41 Belarmino 1984, p. 23. Afirma a continuación que la Luna es el mayor astro después del Sol porque lo dicen los Padres y porque "aparece a los sentidos mayor sin comparación", a pesar de la opinión de los astrónomos de que es el cuerpo celeste menor después de Mercurio. Sobre los tamaños de los astros conocidos en la Antigüedad y la Edad Media, cf. Van Helden 1985, pp. 27-32, 50 .

42 Belarmino 1984, p. 21; cf. la nota 39, pp. 42 y sig. Los matemáticos jesuitas podían condescender con la ocurrencia de Belarmino de que los movimientos de los astros eran libres e irregulares, pero no podrían predecirlos sin reducir la irregularidad aparente a movimientos circulares y uniformes, como enseñaba el Almagesto (Ptolomeo 1984, III .3, p. 216).

43 Lo que lo lleva a decir, contra el principio de relatividad visual conocido por los escolásticos del siglo XIV, que "se experimenta cla-

\section{BIBLIOGRAFÍA}

Belarmino, Roberto (1984), The Louvain Lectures (Lectiones Lovanienses) of Bellarmine and the Autograph Copy of his 1616 Declaration to Galileo, U. Baldini y G. V. Coyne (eds.). Ciudad del Vaticano, Vatican Observatory Publications.

Beltrán Marí, Antonio (2006), Talento y poder. Historia de las relaciones entre Galileo y la Iglesia católica, Pamplona, Laetoli. ramente que la Tierra está firme y que el ojo no se engaña cuando juzga que el Sol se mueve, del mismo modo que no se equivoca cuando juzga que la Luna y las estrellas se mueven. Baste esto por ahora". Carta a Foscarini, en Galileo, Opere XII, p. 172.

44 Belarmino 1984, p. 39, n. 91.

45 Todavía en 1618 trata de conseguir que F. Cesi le dé una solución al enigma de cómo las estrellas pueden ir a su aire y comportarse como si estuviesen en una esfera. Al parecer, en el casi medio siglo transcurrido ni estudió a Copérnico ni se dejó aconsejar por Clavio y sus discípulos matemáticos y seguía creyendo que los astrónomos asignan una sola esfera a cada astro errante más otra a las fijas. Tras señalar que "esta invención mía no es satisfactoria ni para los planetas ni para el firmamento de las estrellas", eso no le preocupa mucho, pues confía en que "cuando lleguemos al cielo, lo comprenderemos todo en un instante" (Blackwell 1991, pp. 43-44).

46 Belarmino, 1984, p. 21. No obstante sus dudas, se mostró más fundamentalista que el propio Concilio de Trento, cuya Sesión IV (8-IV-1546) emitiera el Decreto sobre la interpretación de las escrituras: "en materia de fe y moral,... nadie osará interpretar las Sagradas Escrituras en contra del sentido de la Santa Madre Iglesia... o contra el acuerdo unánime de los Padres..." Sin embargo Belarmino se opuso a las interpretaciones de Agustín, Zúñiga, Foscarini y Galileo en el sentido de que los pasajes aparentemente sobre astronomía (de la que no se ocupa la Biblia) se acomodaban al sentido común de antaño, indicando que si bien por el tema (ex parte objecti) el heliocentrismo no afecta a cuestiones de fe y moral, por quien lo dice (ex parte dicentis; esto es Dios), el movimiento del Sol es cuestión de fe. Blackwell 1991, pp. 183 y 266.

47 A. Oreggi, De Deo uno, 1629, pp. 193-95; citado en Beltrán 2006, p. 515.

48 J. B. Morin, Famosi et antiqui problematis de Telluris motu vel quiete, París, 1631; cf. Galileo, Opere VII, pp. 549 y sigs., esp. 555.

49 El escepticismo filosófico de Oresme lo llevó a defender la incertidumbre del conocimiento científico y la seguridad del teológico: "Only faith could furnish us with certainty. Experience and natural reason could not determine causal truths about the physical world... But although he eroded confidence in our ability to determine natural causal truths, Oresme refrained from invoking God and theology to discredit arguments in natural philosophy. Rather he used his profound knowledge of Aristotelian science and mathematics to undermine the claims for certainty in natural philosophy" (Grant 1988, pp. 39-40); al revés que Belarmino y Barberini quienes invocaron sólo la teología con olvido de la argumentación científica.

Bianchi, Luca (2000), "Interventi divini, miracoli e ipotesi soprannaturali nel Dialogo di Galileo". En: Canziani, G., Granada, M. A. y Zarka Y. C. (eds.), Potentia Dei. L'onnipotenza divina nel pensiero dei secoli XVI e XVII, Milán, Franco Angeli, pp. 239-251.

Bianchi, Luca (2001), “Agostino Oreggi, qualificatore del Dialogo, e i limiti della conoscenza scientifica". En: Montesinos, J. y Solís, C. (eds.), pp. 575-84. 
Blackwell, Richard J. (1991), Galileo, Bellarmine, and the Bible, Notre Dame, University of Notre Dame Press.

Buridán, Jean (1942), Quaestiones super libris quattor de caelo et mundo, E. Moody (ed.). Cambridge, Mass, The Mediaeval Academy of America. Hay reediciones hasta 2012.

Buridán, Jean (2000), Summulae de Dialectica, G. Klima (ed.). New Haven, Yale University Press.

Clagett, Marshall (1959), The Science of Mechanics in the Middle Ages, Madison, The University of Wisconsin Press.

Clagett, Marshall (1968), Nicole Oresme and the Medieval Geometry of Qualities and Motions, Madison, The University of Wisconsin Press.

Clavius, Christoph (1611), Commentarium In Sphaeram loannis de Sacro Bosco \& Astrolabium. En Christophori Clavii Opera Mathematica, III, Moguntiae, Antonii Hierat, excudebat Reinhardus Eltz [loannes Volmari].

Copérnico, Nicolás (1973), Opera omnia, 4 vols. Varsovia, Officina Publica Libris Scientificis Edendis.

Crombie, Alistair C. (1959), The History of Science from Augustine to Galileo. Nueva York, Dover Publications. Hay traducción española, Historia de la ciencia: De San Agustín a Galileo, Madrid, Alianza, 1974

Denifle, Henricus (1889), Chartularium Universitatis Parisiensis, tomo I. París, Ex typis Fratrum Delalain.

Funkenstein, Amos (1986), Theology and the Scientific Imagination from the Middle Ages to the Seventeenth Century, Princeton University Press.

Galilei, Galileo (1890-1909), Le opere di Galileo Galilei, Antonio Favaro (ed.), 20 vols. Florencia, G. Barbèra.

Grant, Edward (1962), "Late Medieval Thought, Copernicus, and the Scientific Revolution", Journal of the History of Ideas, XXIII (2), pp. 197-220.

Grant, Edward (1988), "Nicole Oresme on Certitude in Science and Pseudo-Science”, en Soufrin, P. y Segonds, A. P. (eds.), Nicolas Oresme: Tradition et innovation chez un intellectuel du XIVe siècle, París, Les Belles Lettres, pp. 31-43.

Grant, Edward (1993), "Jean Buridan and Nicole Oresme on Natural Knowledge", Vivarium, XXXI (1), pp. 84-105.

Klima, Gyula (2009), John Buridan. Oxford University Press.

Lattis, James M (1994), Between Copernicus and Galileo. Christopher Clavius and the Collapse of Ptolemaic Cosmology, The University of Chicago Press.

López Prada, Fernando (2014), Usar la razón para confundir a la razón. La epistemología de Nicolás Oresme, trabajo de fin de Master. Madrid, Facultad de Filosofía, UNED.
Maier, Anneliese (1955), “Die naturphilosophische Bedeutung der scholastischen Impetus-theorien", Scholastik, XXX, pp. 321-43. Traducción inglesa en el Capítulo 4 de S. D. Sargent (ed.), On the Threshold of Exact Science. Selected Writtings of Anneliese Maier on Late Medieval Natural Philosophy, Filadelfia, University of Pennsylvania Press, 1982, pp. 77-102.

Marx, Karl (1852), "Der achtzehnte Brumaire des Louis Bonaparte", Die Revolution, Eine Zeitschrift in zwanglosen Heften von Joseph Weydemeyer, Nueva York.

Montesinos, José y Solís, Carlos (eds.) (2001), Largo campo di filosofare. Eurosymposium Galileo 2001. La Orotava, Fundación Canaria la Orotava de Historia de la ciencia.

Oresme, Nicolás (1952), Livre de divinacions y Tractatus contra iudiciarios asrtronomos. En: Coopland, George William (ed.), Nicole Oresme and the Astrologers, Liverpool, Harvard University Press, pp. 50-121 y pp. 123-41.

Oresme, Nicolás (1966), De proportionibus proportionum and Ad pauca respicientes, Edward Grant (ed.). Madison, The University of Wisconsin Press.

Oresme, Nicolás (1968), Le livre du ciel et du monde, A. D. Menut y A. J. Denomy (eds.). Madison, The University of Wisconsin Press.

Oresme, Nicolás (1971), Nicole Oresme and the Kinematics of Circular Motion: Tractatus de commensurabilitate vel incommensurabilitate motuum celi, E. Grant (ed.). Madison, The University of Wisconsin Press.

Oresme, Nicolás (1973), "Quodlibeta” En: B. Hansen (ed.), Nicole Oresme and the marvels of nature: a critical edition of his Quodlibeta with English translation and commentary, Princeton University Press.

Oresme, Nicolás (1976), "Quaestio contra divinatores horoscopios", Archives d'Histoire Doctrinale et Littéraire du Moyen Age, París, Vrin, pp. 201-310.

Oresme, Nicolás (2007), De visione stellarum, D. Burton (ed.). Leiden, Brill.

Piché, David (1999), La condamnation parisienne de 1277, nouvelle édition du texte latin, traduction, introduction et commentaire par David Piché avec la collaboration de Claude Lafleur. París, Vrin.

Ptolomeo, Claudio (1984), Almagest, G. J. Toomer (ed.). Londres, Duckworth

Solís, Carlos (2001), “La cosmología oculta de Galileo". En: Montesinos, José y Solís Santos, Carlos (coords.), Largo campo di filosofare Eurosymposium Galileo 2001, pp. 357-379.

Van Helden, Albert (1985), Measuring the Universe. Cosmic Dimensions from Aristarchus to Halley, The University of Chicago Press.

Weijers, Olga (2005), Le travail intellectuel à la Faculté des arts de Paris: textes et maîtres (c. 1200-1500), Turnhout, Brepols.

Zúñiga, Diego de (1584), In Job commentaria, Toledo, Johannes Rodericus. 